Université de Ljubljana*

\title{
LES JE GIGOGNES DU ROMAN CÉLINIEN
}

\section{INTRODUCTION}

Le présent article se penchera sur des aspects narratologiques des trois derniers romans de l'écrivain français Louis-Ferdinand Céline (1894-1961), D'un château l'autre (1957), Nord (1960) et Rigodon (publication posthume en 1964). Les romans, que la tradition critique solidement établie réunit en trilogie allemande, ${ }^{1}$ présentent l'aboutissement des recherches poétiques de l'écrivain tant au niveau du style qu'au niveau des techniques narratives. L'analyse qui s'appuiera pour l'essentiel sur le modèle narratologique de Gérard Genette (Genette 1972, 1983) se centrera sur différentes valeurs du je célinien :

- je comme instance(s) narrative(s),

- je comme foyer(s) de perception,

- je comme personnage(s) romanesque(s).

\section{L'IDENTITÉ DU JE CÉLINIEN}

La linguistique identifie le pronom personnel je au sujet producteur du discours. Or cette identification, parfaitement légitime dans le cas des échanges langagiers " de tous les jours ", ne saurait s'appliquer au discours littéraire. Il est évident qu'il faut, dès l'entrée en matière, distinguer le je-énonciateur « réel » du je-narrateur « fictif ». Ferdinand, Ferdine, Louis, Céline ou Destouches qui se cachent derrière les je de l'univers romanesque de la trilogie allemande ne "sont » aucunement l'écrivain français Louis-Ferdinand Destouches dit Céline. Les instances énonciatives du discours littéraire sont dédoublées en instances extratextuelles et intratextuelles. Du côté de la production discursive, l'auteur en chair et en os s'oppose au narrateur qui n'est qu'un élément de l'univers romanesque. Le dédoublement analogue se produit aussi du côté de la réception. Si la production littéraire de l'auteur est destinée au lecteur effectif « extratextuel», le narrateur s'adresse au narrataire appartenant lui aussi à l'univers intratextuel.

\begin{tabular}{|llll|} 
NIVEAU EXTRATEXTUEL : & auteur & $\rightarrow$ & lecteur \\
\hline NIVEAU INTRATEXTUEL : & narrateur & $\rightarrow$ & narrataire \\
\hline
\end{tabular}

\footnotetext{
*Adresse de l'auteur : Filozofska fakulteta, Oddelek za romanske jezike in književnosti, Aškerčeva 2, 1000 Ljubljana, Slovénie. Mél: gregor.perko@guest.arnes.si

${ }^{1}$ C'est le nom qu'emploie, entre autres, Henri Godard dans la Préface et l'appareil critique de l'édition des romans dans la Pléiade. Céline n'a jamais désigné sous ce nom les trois romans en question. Ses projets étaient plus ambitieux et envisageaient un ensemble plus grand relatant encore le séjour de l'écrivain au Danemark.
} 
Force est d'ajouter que même ce schéma dédoublé s'avère trop simplifié pour de nombreux textes littéraires. C'est par exemple le cas des romans "dialogiques" (cf. Bakhtine 1977) ou bien, comme nous le verrons plus loin, celui des textes à « récits enchâssés ».

Le rapport entre l'auteur et le narrateur semble s'obscurcir lorsqu'il s'agit d'un texte littéraire à visée autobiographique. En effet, le texte autobiographique postule l'identité entre le narrateur, l'auteur et le personnage principal. Or, le caractère autobiographique d'un récit n'a pas d'incidence sur une analyse narratologique qui ne franchit pas le seuil du texte. Le $j e^{2}$ n'établit que l'identité entre les deux instances narratives au plan intratextuel, à savoir le narrateur et le personnage. Rien dans le texte lui-même ne permet cependant d'établir l'identité entre le narrateur et l'auteur. Ce n'est que la confrontation entre l'univers intratextuel et la "réalité » extratextuelle à laquelle appartient l'auteur qui nous le permet. En réalité, cette identité n'est garantie que par une affirmation explicite dans le texte ou le paratexte qu'atteste le nom de l'auteur ou son pseudonyme. ${ }^{3}$

Étant donné que le caractère autobiographique relève plus d'une question " juridique " que d'une question narratologique ou linguistique, notre analyse ne fera pas entrer en ligne de compte la dimension autobiographique des romans de Céline. Le je de l'auteur sera en conséquence écarté de notre étude.

\section{LA VOIX DES $J E$ NARRATEURS OU LA NARRATION MISE À NU}

L'histoire de la trilogie n'est pas facile à résumer et se présente comme une coexistence de deux récits différents dont l'un raconte la traversée de l'Allemagne effectuée par Céline, sa femme Lili et leur chat Bébert pendant les dernières années de la Seconde Guerre mondiale et l'autre la "vie de Céline " à Meudon dans la deuxième moitié des années cinquante. D'un côté, les images d'un pays apocalyptique et cauchemardesque submergé par des soldats en déroute, des réfugiés et des blessés, de l'autre côté, le portrait d'un écrivain-médecin aux prises avec les patients, les éditeurs, les journalistes, l'actualité politique et littéraire, mais avant tout avec l'oeuvre qu'il s'escrime à écrire. Les deux récits s'entrecoupent sur toute la longueur de la trilogie créant une impression du désordre et de la désorganisation.

Au premier abord, il semblerait que la différence entre ces deux histoires ne soit que de l'ordre temporel : les histoires racontent deux époques d'une même existence, l'une étant d'une dizaine d'années postérieure à l'autre. Mais une telle interprétation ne tient pas compte de la spécificité de leur relation mutuelle ni par conséquent de leurs fonctions respectives dans la structure narrative. La valeur principale du récit de Meudon (« récit ultérieur ») se révèle être l'évocation du lieu,

\footnotetext{
${ }^{2}$ L'emploi de je n'est nullement la marque exclusive du texte autobiographique. Il est évident que tous les romans écrits à la première personne ne sont pas des autobiographies et que toutes les autobiographies ne sont pas écrites "à la première personne " (par exemple, Autobiographie de Frederico Sánchez de Jorge Semprun /1978/ ou Frêle bruit de Michel Leiris /1976/ qui utilisent respectivement $t u$ et $i l$.)

${ }^{3}$ Sur les difficultés d'une définition linguistique ou narrative de l'autobiographie, voir Lejeune 1975.
} 
du temps, des circonstances et de l'acte de la narration. ${ }^{4}$ Le lecteur se trouve en présence d'un personnage-écrivain qui s'applique à rassembler ses souvenirs, à raconter une histoire, à la mettre en forme, bref, à écrire un roman, mais qui en est constamment empêché : la pression écrasante de souvenirs disparates s'annulant à tour de rôle, la fièvre, les visites d'importuns, les soucis matériels, etc. ${ }^{5} \mathrm{Ce}$ qui peut paraître de prime abord comme une suite de manifestations ou d'intrusions plus ou moins " incohérentes" du narrateur dans le récit doit en réalité être considéré comme un récit autonome et organisé avec sa propre diégèse ${ }^{6}$ (Meudon dans les années 1954-1960, le pavillon de Céline), ses propres personnages, son propre enchaînement d'événements et sa propre valeur thématique. Le personnage principal de ce récit est un écrivain qui raconte " sous nos yeux » le récit de sa traversée de l'Allemagne vers la fin de la Seconde Guerre. Les deux récits ne sont pas simplement " apposés » l'un à l'autre, puisque le récit de Meudon " enchâsse » celui de la traversée de l'Allemagne. L'acte de narration du récit « enchâssé » est donc un événement raconté dans le récit "enchâssant » et le narrateur du récit « enchâssé » est un personnage du récit « enchâssant ».

La notion traditionnelle "d'enchâssement des récits 》 a été systématisée par G. Genette. Sa théorie de la voix (Genette 1972 : 1983) distingue plusieurs niveaux narratifs. Selon ce modèle, les événements racontés par le récit de Meudon (récit « enchâssant ») sont qualifiés de diégétiques ${ }^{7}$ et les événements racontés par le récit de l'aventure allemande (récit " enchâssé ») de métadiégétiques. Le récit métadiégétique est à un niveau narratif supérieur à celui du récit diégétique. Le narrateur du récit métadiégétique qui est un personnage du récit diégétique est appelé diégétique (ou intradiégétique). ${ }^{8}$ Dans le cas des romans de Céline où le narrateur est incontestablement le " héros » de son récit, nous pouvons parler du narrateur autodiégétique. Le narrateur du récit diégétique (récit de Meudon) n'étant inclus dans aucun récit est dit extradiégétique et ne doit pas être confondu avec l'auteur.

Le schéma " classique » d'un récit à la première personne comportant le plus souvent un je-narrateur qui est à la fois extradiégétique et homodiégétique se

\footnotetext{
${ }^{4}$ Nous parlons bien entendu de la narration « fictive » qu'il s'agit de distinguer de la genèse « réelle » des romans.

${ }^{5}$ Plus qu'un récit de l'écriture, les romans de Céline sont des récits des dificultés, voire de l'impossibilité de l'écriture.

${ }^{6} \mathrm{La}$ notion de diégèse désigne « l'univers spatio-temporel désigné par le récit 》 (Genette $1972: 280$ ).

${ }^{7}$ L'adjectif diégétique désigne tout ce qui appartient à l'histoire.

${ }^{8}$ La théorie de Genette, se basant sur la relation entre le narrateur et l'histoire qu'il raconte, permet d'éviter des écueils qu'ont rencontrés la plupart des modèles narratifs antérieurs. Les théories plus traditionnelles utilisent le plus souvent la catégorie de la personne: on parle des récits à la première, à la troisième et même à la deuxième personne. Ces termes gramaticaux sont inadéquats parce qu'ils occultent l'essentiel du problème qui ne réside pas dans l'utilisation d'une forme verbale à la place d'une autre. Ce qui importe c'est de savoir si le narrateur est un personnage de l'histoire qu'il raconte ou si, au contraire, il en est absent. En outre, tout récit est en fait écrit à la première personne, le narrateur étant toujours le sujet de l'énonciation qu'il fait.
} 
complique considérablement. Le narrateur extradiégétique fait figurer dans le récit son " image spéculaire » qui réfléchit à l'intérieur du récit ce qui est supposé resté dehors : le processus de la narration (Dällenbach 1977: 100-122). Derrière le je-narrateur de la trilogie se cachent en fait deux instances narratives : un je extradiégétique et un je diégétique. Le $j e$ extradiégétique est en même temps homodiégétique (plutôt autodiégétique), puisqu'il est le personnage (plutôt le héros)

\begin{tabular}{|l|c|c|}
\hline & NARRATEUR & PERSONNAGE/HÉROS \\
\hline NIVEAU DU RÉCIT MÉTADIÉGÉTIQUE & \multicolumn{2}{|c|}{$\Leftrightarrow$} \\
\hline NIVEAU DU RÉCIT DIÉGÉTIQUE & $j e^{2}$ \\
\hline NIVEAU EXTRADIÉGÉTIQUE & $j e^{l}$ & $j e^{l}$ \\
\hline
\end{tabular}

de son propre récit. Et ce je-narrateur-héros est à son tour le narrateur du récit métadiégétique dans lequel il est le personnage principal.

En termes plus simples : Céline en tant que narrateur extradiégétique $\left(j e^{l}\right)$ nous montre " lui- même " $\left(j e^{1}\right)$ en train d'écrire un roman où il $\left(j e^{2}\right)$ relate ses propres aventures passées $\left(j e^{2}\right)$. Un même personnage assume donc deux fonctions identiques à deux niveaux différents. ${ }^{9}$

L'impression de " confusion » sentie par le lecteur est due au fait que le récit passe d'un niveau narratif à l'autre sans que ce passage soit nettement marqué. Les indicateurs les plus sûrs qu'un récit diégétique devient métadiégétique sont en règle générale des procédés syntaxiques (changement de personne grammaticale ou de genre...) ou lexicaux (changement de nom propre...). En raison du caractère homodiégétique des deux narrateurs de la trilogie, ces marques font bien évidemment défaut. Le je extradiégétique cède la place et la responsabilité de mener le récit au je diégétique qui n'apparaît plus comme un personnage du récit diégétique mais seulement comme le narrateur de son propre récit. Ce qui nous avertit du passage d'un niveau narratif à l'autre, c'est le changement de la diégèse : l'univers spatio-temporel du récit diégétique s'efface au profit de l'univers spatio-temporel du récit métadiégétique. ${ }^{10}$

Nous avons déjà mentionné que les deux récits s'entrecoupent et s'entremêlent tout au long de la trilogie. En effet, le récit diégétique ne se réduit pas à un simple récit-cadre qui aurait introduit puis clos le récit métadiégétique. Voyons maintenant, dans les grandes articulations, comment ces niveaux se manifestent dans les trois romans.

Le récit diégétique occupe presque la moitié de D'un château l'autre, ce qui dépasse largement les proportions dans les deux autres romans. Jusqu'à la page 102,11

\footnotetext{
${ }^{9}$ La situation où un narrateur extradiégétique devient lui-même intradiégétique, appelée aussi situation de double narrateur, est à notre connaissance rarissime. Voir aussi Genette (1972 : 239).

${ }^{10} \mathrm{La}$ « mue » du récit est de temps en temps signalée explicitement. Dans Rigodon, par exemple, après un dialogue avec le colonnel Cambremousse à Meudon, le héros-narrateur annonce ainsi la reprise du récit métadiégétique : «Et je me remets au travail... » (Rigodon, p. 725).

${ }^{11}$ Toutes les pages indiquées renvoient à la pagination de la Pléiade (voir Bibliographie).
} 
c'est l'univers de Meudon dans les années cinquante qui est mis en scène. Le héros-écrivain évoque ses souvenirs (enfance, emprisonnement au Danemark) qui ne s'organisent pas dans un récit proprement dit. Son travail d'écrivain est constamment contrarié par les soucis matériels, par les visites de malades ou de rares amis, par les disputes avec ses éditeurs ou avec des journalistes. Lors d'une visite qu'il rend à une de ses malades, Mme Niçois, il succombe à un accès de paludisme qui provoque des hallucinations (p. $70 s q$.) : Il se trouve devant l'apparition d'un bateau-mouche avec le nocher Caron et Le Vigan..$^{12}$ Les souvenirs que fait naitre cette apparition ne se distinguent, au début, en rien des autres souvenirs évoqués. Ils ne paraissent être qu'une nouvelle « digression » causée par un délire fiévreux. Mais après la page 102, les contours de ce qui sera le récit métadiégétique se précisent peu à peu. Jusqu'à la page 117, le récit subsiste dans une zone d'ombre où l'univers de Meudon s'efface peu à peu devant le décor de Sigmaringen en 1944. Après le départ de Mme Niçois à l'hôpital (pp. 145-147), le récit métadiégétique démarre pour être brièvement interrompu par un accès de découragement (pp. 194-195). Quelques pages avant la fin du roman (p. 291), le récit de Sigmaringen est terminé. Dans les pages qui suivent, le héros-narrateur prophétise le sort qui attend le livre " terminé », commente des actualités et de nouveau c'est Mme Niçois qui lui rend visite. Ajoutons que Mme Niçois est un personnage important du récit diégétique. Son destin (maladie, envoi à l'hôpital, sortie de l'hôpital) forme une petite histoire qui accompagne le procès (fictif) de la rédaction de la " chronique » de Sigmaringen. Le récit diégétique n'est donc pas une simple suite d'intrusions du narrateur mais bien un récit où ses personnages assument leur existence dans un univers spatio-temporel déterminé.

Nord commence (p. 303) à Meudon par ce qu'on pourrait appeler " réflexions générales ». Mais à la différence du roman précédent, le récit des aventures guerrières démarre assez vite. Un court dialogue (pp. 304-305) qui éveille le souvenir d'une vieille dame que Céline a connue pendant la guerre suffit pour que le récit se transpose, après quelques va-et-vient entre les deux niveaux, dans le décor de Baden-Baden en juillet 1944. Il est à souligner que les retours au récit diégétique sont moins fréquents dans Nord que dans les deux autres romans. Quelques exemples : a ux pages 502-509, le récit du séjour à Zornhof est interrompu par des commentaires sur l'actualité littéraire et politique et par la visite de Roger Nimier ; à la page 618, c'est l'exposé de son credo artistique ; aux pages 649-654, Céline rend visite à l'éditeur Brottin. La clôture du roman démontre bien sa position médiane dans la trilogie : le récit n'est pas ramené au niveau diégétique mais reste « suspendu » sur une discussion entre deux personnages du récit métadiégétique, Harras et Göring (pp. 706-707).

La structure de Rigodon est analogue à celle des deux premiers romans. De nouveau, le récit métadiégétique éprouve des difficultés à démarrer et "s'arrête " toutes les fois que le héros-narrateur est dérangé par des vistes de journalistes, par des brouilles avec les éditeurs ou les amis. Le va-et-vient entre les deux niveaux

12 Robert Le Vigan, acteur français (1900-1972), était compagnon des Céline pendant une partie de la traversée de l'Allemagne. Il figure aussi dans Nord et Rigodon. 
s'intensifie vers la fin, surtout après l'épisode où, pendant un raid aérien à Hanover, le héros est atteint par une brique "en pleine caboche » (p. 823). Comme si la défaillance physique du héros "métadiégétique » qui a résulté du choc requérait une plus grande présence du narrateur diégétique. La trilogie se termine au niveau diégétique par l'évocation prophétique et grotesque du "péril jaune ", une des obsessions majeures de Céline.

La distinction entre les deux niveaux narratifs et les deux narrateurs nous a permis d'expliquer la complexité de la structure narrative de la trilogie qui rompt avec la linéarité de l'expression romanesque " traditionnelle ». Céline refuse l'illusion " réaliste " des $\mathrm{XIX}^{\mathrm{e}}$ et $\mathrm{XX}^{\mathrm{e}}$ siècles qui nous faisait croire à un récit romanesque idéal issu de nulle part. L'histoire ne peut pas se raconter toute seule, indépendamment du narrateur et de la situation dans laquelle celui-ci travaille. $\mathrm{Si}$ le narrateur est gâteux, fiévreux, délirant, tracassé par des soucis financiers et l'insuccès de ses oeuvres et si, de plus, il est constamment dérangé dans son travail, le récit qu'il rédige, doit s'en ressentir. "L'entreprise romanesque est ici tout entière marquée par une conscience aiguë de sa dualité d'histoire et de narration " (Godard 1985 : 305).

La subjectivité des je-narrateurs est omniprésente et omnipotente et perturbe forcément la réalité perçue. Les chroniques, comme Céline désignait les romans de la trilogie, démontrent l'impossibilité d'enregistrer fidèlement les faits puis de les raconter dans leur " ordre » logique et chronologique. Les je céliniens se montrent incapables, dans leur état psycho-somatique et dans les circonstances du moment, de mener à bien une telle entreprise. Plutôt qu'une oeuvre en construction, les romans de Céline nous apparaissent comme une oeuvre en déconstruction.

\section{LES JE CÉLINIENS COMME ASSISES DE LA PERSPECTIVE NARRATIVE}

La question de la perspective narrative peut se résumer ainsi : "Qui perçoit dans le roman? ». La perspective détermine la quantité de savoir sur l'univers romanesque que véhicule un roman. Cette information peut parvenir au lecteur sans aucun filtrage ou sélection ou bien partielle, restreinte, filtrée par les capacités de connaissance d'un ou de plusieurs personnages du roman. G. Genette (1972 : 183-224; 1983 : 43-48) distingue les focalisations zéro (perspective centrée sur le narrateur), interne (perspective centrée sur un personnage) et externe (perspective située à l'intérieur de l'univers diégétique mais hors de tout personnage). Vu la complexité de la structure narrative de la trilogie, la réponse à la question de la perspective n'est pas facile.

Le récit diégétique est ordonné par la narration simultanée. Aucune distance temporelle ne semble séparer les événements de ce récit du moment de leur narration. Le présent qui domine largement signale les événements racontés comme relevant du nunc. La période du temps à laquelle réfère le procès est souvent référentiellement très étroite, ce qui crée l'illusion que le héros-narrateur (qui est " sur scène ») décrit les événements " en même temps » qu'il les assume ou les produit. La simultanéité de la narration et l'identité entre le narrateur et le héros 
entraînent l'équivalence du savoir du je-narrateur et du je-héros : le narrateur ne dit rien de plus que ce que perçoit et sait le héros. Le récit diégétique est donc un « pur » récit à focalisation interne.

Le récit métadiégétique raconte les événements qui sont d'une décennie antérieurs au moment de la narration. Les événements étant révolus, le narrateur en connaît parfaitement les suites et les conséquences. La différence entre le je-héros et le je-narrateur provoque une sorte de frottement entre les capacités de connaissance du héros et le savoir présent du narrateur. La possibilité d'une application rigoureuse de la focalisation interne s'en trouve réduite. L'information que détient le héros au moment de l'histoire est de temps à autre complétée par les informations que le héros devenu narrateur obtient ultérieurement. Ces informations " complémentaires » excédant l'univers spatio-temporel du récit ne peuvent être le fait du je-héros mais celui du je-narrateur. G. Genette (1983 : 51-52) parle de focalisation sur le narrateur. Cette perspective qui est le propre du récit autodiégétique est par définition limitée puisque le je ne «peut » rapporter que les informations dont il est responsable, soit en tant que héros soit en tant que narrateur.

Dans la trilogie allemande, la focalisation sur le narrateur « se réduit » le plus souvent à la focalisation interne et diffère donc radicalement de la perspective illimitée d'un narrateur omniscient : la somme d'informations avec laquelle opère le narrateur extradiégétique n'excède qu'exceptionnellement les connaissances du héros métadiégétique " relayées » par le narrateur diégétique. Le château de Sigmaringen et le séjour des réfugiés, par exemple, ne sont jamais décrits dans leur totalité. C'est la présence du héros qui crée la possibilité de les "mettre en scène ». Nous ne savons rien de ce que fait Pétain à Sigmaringen et la partie du château qu'il habite n'est jamais décrite du simple fait que le héros n'y a jamais mis les pieds. Pétain n'apparaît qu'une seule fois : c'est l'évocation parodique d'une promenade du maréchal et de ses ministres dont faisait par hasard partie le héros. À part cela, le narrateur se borne à quelques ragots ou autres allusions menues que le héros a pu entendre au cours de ses conversations. De même, nous ne savons rien d'autre sur les pensées ou les sentiments des autres personnages figurant dans la trilogie que ce que le héros peut conclure des propos explicites de ces personnages, de leur comportement ou de quelque autre manifestation de réalités psychiques. ${ }^{13}$

Le récit métadiégétique est dominé par l'imparfait ${ }^{14}$ et le présent. Le rôle premier de l'imparfait est de marquer que nous franchissons le seuil du récit métadiégétique. L'imparfait donne l'instruction que le point de référence ( $c f$. Molendijk 1985) à partir duquel les événenements sont perçus est situé dans l'époque passée :

\footnotetext{
${ }^{13}$ Il peut sembler que les personnages céliniens n'aient pas assez « d'individualité » et qu'ils n'existent que par et pour le héros-narrateur. Plutôt qu'une tare, ce prétendu manque de psychologisme mérite d'être considéré comme une conséquence inévitable de la perspective narrative « réduite » appliquée par Céline.

${ }^{14}$ La place impartie à notre article ne nous permet pas de se consacrer à une analyse plus approfondie des temps verbaux dans les trois romans. Nous laisserons complètement de côté le passé composé et le passé simple ainsi que les « temps de l'antériorité ».
} 
« Révolte... pas au Bas-Meudon ! non !... à Siegmaringen ${ }^{15}$ je bats la campagne, je vous promène.. soit !... je rassemble mes souvenirs historiques... que je me trompe pas !... nous y voilà !... Siegmaringen... l'état du moral !... pas fameux !... malgré les appels à la « conscience combattante » de « l'Europe Unie » flasque ! aussi flasque qu'aux jours de maintenant, malgré les appels de Dulles, Coty, Lazare, Youssef, le Pape... mou, mou, mou moral !... les « certitudes en la Victoire »... qu'elle était là, et patati !... réchauffaient personne ! ça mouftait pas, mais pensait bas !» (D’un château l'autre, pp. 117-118)

Non seulement l'imparfait dénote des procès continus, non bornés, mais il permet encore de se placer à l'intérieur de la diégèse du récit métadiégétique. L'imparfait met en place un point de vue subjectif ( $c f$. Benveniste 1966a ; Barceló/Bres 2006 : 55-57) qui va de pair avec la focalisation interne : les événements sont perçus à travers les yeux et les oreilles du je-héros métadiégétique.

Le récit métadiégétique, qui commence à l'imparfait, glisse régulièrement au présent. $\mathrm{Au}$ fur et à mesure que le récit métadiégétique prend plus d'ampleur, notamment dans Nord et Rigodon, les passages de l'imparfait au présent se font plus rapides :

«Que je revienne à mon histoire... Mme von Secktnous faisait ses adieux... son petit souvenir, l'éventail... voilà !... le lendemain matin comme prévu, à l'aube, Schulze frappe... l'hôtel dort... mais nous sommes prêts, Bébert dans son sac... nos deux valises et en avant! » (Nord, p. 330)

L'emploi du présent réduit le décalage temporel entre la perception et la narration, ce qui crée une impression d'immédiateté de la perception. Cette impression est accentuée, entre autres procédés stylistiques, par le recours à ce que Céline appelle « [son] décor sonore » (Rigodon, p. 829). Le récit est ponctué d'onomatopées imitant des explosions de bombes, des vrombissements d'avions, des sirènes, des bruits de trains, etc :

« ...et que ça se ramasse !..hop ! et se bat, s'empoigne ! boum !... braoum ! même les tout-petits font braoum !... imitent ! font les bombes comme sur Berlin ! là-bas ! et zzz ! comme les avions !... aussi, ils savent ! braoum ! et zzz !... que tout culbute ! mômes et les briques! zzz! et en avant ! » (Nord, 675)

Le décor sonore qui nous parvient par la voix des je céliniens traduit l'expérience $\mathrm{du}$ monde dans toute sa matérialité car le bruit ne s'absorbe pas dans la signification. Il incite le lecteur à « écouter » le texte avant de le « lire ». Les mots sont ainsi détournés de leur valeur dénotative pour éclater dans l'immédiat d'une sensation auditive.

${ }^{15}$ Il ne s'agit pas de faute d'orthographe : c'est la « graphie » de Sigmaringen dans D'un château l'autre. 


\section{CONCLUSION}

L'analyse narratologique des trois derniers romans de Louis-Ferdinand Céline a montré que la réponse à la question de savoir à quoi réfère le je du discours romanesque ne saurait recevoir de réponse simple et univoque. Un roman à la première personne (voire une autobiographie) peut comporter plusieurs instances narratives, plusieurs foyers de perception et plusieurs héros, tous désignés par un seul et même pronom : je.

Les évenements racontés dans la trilogie allemande de Céline s'organisent sur deux niveaux narratifs dont l'un, le récit diégétique, intègre l'autre, le récit métadiégétique. Un je-héros métadiégétique est pris en charge par un je-narrateur diégétique étant en même temps un je-héros diégétique pris à son tour en charge par un je-narrateur extradiégétique. Un je peut en cacher un autre.

\section{Bibliographie}

\section{A. Romans étudiés}

CÉLINE, Louis-Ferdinand (1974) Romans II. Paris : Gallimard (Bibliothèque de la Pléiade) [le volume contient D'un château l'autre, Nord et Rigodon].

\section{B. Références}

BAKhtine, Mikhail (1977) Le marxisme et la philosophie du langage. Paris : Minuit.

BARCELÓ, Gérard Joan/Jacques BRES (2006) Les temps de l'indicatif en français. Paris : Ophrys. Benveniste, Émile (1966) Problèmes de linguistique générale. Paris : Gallimard.

BENVENISTE, Émile (1966a) «Les relations de temps dans le verbe français. » In : Benveniste (1966), 237-257.

BeNVENISTE, Émile (1966b) « De la subjectivité dans le langage. » In : Benveniste (1966), 258-266.

DällenbaCh, Lucien (1977) Le récit spéculaire. Essai sur la mise en abyme. Paris : Seuil. Genette, Gérard (1972) Figures III. Paris : Seuil.

- (1983) Nouveau discours du récit. Paris : Seuil.

- (1991) Fictions et dictions. Paris : Seuil.

GLowinski, Michal (1987) « Sur le roman à la première personne. » Poétique 72, 497-506.

GodARD, Henri (1985) Poétique de Céline. Paris : Gallimard.

Ifri, Pascal A. (1987) « Focalisation et récits autobiographiques. » Poétique 72, 483-495.

Jost, François (1975) « Le je à la recherche de son identité. » Poétique 24, 479-487.

Kerbrat-Orecchioni, Catherine $\left({ }^{4} 1999\right)$ L'énonciation. Paris : Armand Colin.

LeJeune, Philippe (1975) Le pacte autobiographique. Paris : Seuil.

MoLENDIJK, Arie (1985) « Point référentiel et imparfait. » Langue française 67, 78-94.

Perko, Gregor (1997) La trilogie allemande de Louis-Ferdinand Céline : une approche narratologique. Ljubljana : Filozofska fakulteta (mémoire de maîtrise).

Rabatel, Alain (2001) « Fondus enchaînés énonciatifs. Scénographie énonciative et points de vue. » Poétique 126, 151-173. 


\section{Povzetek \\ $J A Z$ ZNOTRAJ JAZA V ROMANIH L.-F. CÉLINA}

Članek prinaša analizo izbranih narativnih značilnosti zadnjih treh romanov francoskega pisatelja Louisa-Ferdinanda Célina (1984-1961): D'un château l'autre (Iz gradu v grad), Nord (Sever) in Rigodon (Rigodon). Kot teoretični okvir služi danes že dobro uveljavljeni naratološki model Gérarda Genetta. Članek se osredotoča na različne vrednosti zaimka je (jaz) in poskuša pokazati, da je tudi v romanu, pisanem v prvi osebi, nujno razlikovati med več pripovednimi instancami, več središči pripovedne perspektive in več junaki oziroma glavnimi osebami, ki se vsi »skrivajo« za zaimkom prve osebe ednine.

Jezikoslovje prvo osebo ednine izenačuje s subjektom izrekanja. Literarni diskurz se takšni izenačitvi izmika. Naratologija že dolgo razlikuje med "resničnim« subjektom izrekanja, to je pisateljem, in pa pripovedovalcem, ki pripada literarnemu diskurzu. V primeru narativno kompleksnejših besedil, kakršni so tudi omenjeni Célinovi romani, je delitev na »pisatelja in "pripovedovalca« nujno dopolniti. Pripoved analiziranih romanov se odvija na dveh ravneh, na diegetski in metadiegetski. Za vsako od ravni je odgovoren eden od pripovedovalcev: izvendiegestki $\left(j a z^{1}\right)$ in diegetski $\left(j a z^{2}\right)$. Diegetski pripovedovalec je hkrati glavna oseba $\left(j a z^{1}\right)$ diegetske pripovedi, v kateri sam "nastopa k kot glavna oseba na metadiegetski ravni $\left(j a z^{2}\right)$. 